\title{
Modelagem Estatística do Algoritmo CSG Aplicada para um Arranjo Adaptativo de Antenas
}

\author{
Javier E. Kolodziej, Orlando J. Tobias e Rui Seara
}

\begin{abstract}
Resumo-Este artigo apresenta um modelo analítico para o algoritmo do gradiente estocástico restrito (CSG). O CSG é utilizado para se determinar os coeficientes ótimos de arranjos de antenas em sistemas celulares, visando maximizar a razão sinal/ruído-mais-interferência (SINR) do sistema. Para a avaliação de desempenho do algoritmo, são consideradas diversas condições de operação, as quais são obtidas através da combinação dos diferentes ângulos de chegada do sinal desejado e dos possíveis interferentes. $O$ fato de contar com um modelo que descreva o comportamento do sistema é de grande utilidade para se obter uma avaliação exaustiva do algoritmo adaptativo nos diferentes cenários de operação. Assim, são derivadas aqui expressões para o momento de primeira ordem do vetor de coeficientes e para a curva de SINR. O modelo é obtido assumindo passos de adaptação pequenos, condição esta que permite uma convergência menos ruidosa. Através de simulações numéricas, é avaliada a precisão do modelo proposto.
\end{abstract}

Palavras-chave-Modelagem estatística, Algoritmo CSG, Arranjo de antenas para comunicações móveis.

Abstract-This paper presents an analytical model for the constrained stochastic gradient (CSG) algorithm. The CSG is used to determine the optimum coefficients of antenna arrays for mobile communications, aiming to maximize the signal-to-interference-plus-noise ratio (SINR) of the system. For the algorithm performance evaluation, several operating conditions are considered by combining a wide variety of arrival-angles for both desired and interference signals. To have an analytical model to describe the system behavior is very useful, since it permits an efficient evaluation of the algorithm performance under several working conditions. Thus, analytical expressions for the first moment of the coefficient vector and the SINR curve are here derived. The proposed model is obtained considering small step-size condition, resulting in a smooth behavior for both the weight and SINR curves. Through numerical simulations, the accuracy of the proposed model is assessed.

Keywords - Statistic models, CSG algorithm, Antenna arrays for mobile communications.

\section{INTRODUÇÃO}

Nos últimos anos, as redes de comunicação sem fio vêm experimentando um aumento crescente no número de usuários,

Javier E. Kolodziej e Rui Seara, LINSE - Laboratório de Circuitos e Processamento de Sinais, Depto. de Eng. Elétrica, Universidade Federal de Santa Catarina, Florianópolis, SC, E-mails: \{javier, seara\}@linse.ufsc.br.

Orlando J. Tobias, LINSE/UFSC e Departamento de Engenharia Elétrica e Telecomunicações, Universidade Regional de Blumenau, Blumenau, SC, E-mail: tobias.oj@ieee.org.

Este trabalho foi parcialmente financiado pela Coordenação de Aperfeiçoamento de Pessoal de Nível Superior (CAPES) e pelo Conselho Nacional de Desenvolvimento Científico e Tecnológico (CNPq). gerando, assim, sérios problemas em ambientes densamente urbanizados, visto que o espectro de freqüências está se aproximando do limite de sua capacidade [1]; nesses casos, atualmente, células setorizadas são utilizadas. Para superar a barreira imposta por um ambiente de espectro limitado é necessário reduzir o tamanho das células aumentando assim a reutilização de freqüências. Por exemplo, em um esquema de sete células por cluster, com três setores de $120^{\circ}$ por célula, a quantidade de freqüências portadoras oferecida a cada setor é $1 / 21$ do total de freqüências disponíveis. Um esquema de três células por cluster incrementa a capacidade por um fator de aproximadamente $7 / 3$. Porém, a distância entre células com co-canais interferentes decresce por um fator de $\sqrt{21 / 9}$, aumentando o nível desse tipo de interferência [2].

Uma estratégia para reduzir tal interferência é utilizar, na estação de rádio base (ERB), arranjos de antenas com diagramas de irradiação conformados segundo a posição dos terminais móveis da própria célula e do co-canal.

Idealmente, toda ERB deveria conhecer a posição de todos os terminais móveis da área, obtendo assim uma solução ótima global. No estado atual, isso não é ainda possível de ser implementado. Nos dias de hoje, tudo o que uma ERB conhece sobre os terminais móveis das células vizinhas é determinado através dos sinais do enlace reverso (uplink) do arranjo. Com essa informação disponível, pode ser usada uma abordagem em que as células vizinhas reduzam a potência transmitida na direção dos terminas móveis do co-canal; assim, a interferência é reduzida globalmente.

Nos sistemas em que a comunicação bidirecional (full-duplex) é realizada através da técnica de divisão de freqüência (FDD - frequency division duplex), um canal é utilizado para o enlace reverso (uplink) e outro próximo para o enlace direto (downlink). Para sinais com freqüências na faixa dos gigahertz, comumente usadas em comunicações celulares, as freqüências portadoras dos enlaces (reverso e direto) estão suficientemente separadas para que o desvanecimento (fading) não afete ambos os enlaces da mesma forma. Em conseqüência, um conhecimento detalhado do desvanecimento do enlace reverso não seria útil na estimação daquele do enlace direto. Porém, as bandas de ambos os enlaces em FDD são geralmente próximas, o que faz com que a geometria do ambiente de propagação seja similar, resultando assim em matrizes de covariância similares [1]. Em [3]-[5], essa propriedade é explorada para o controle do arranjo adaptativo considerando estimações das matrizes de covariância tanto do sinal do enlace direto quanto do sinal interferente (ambas obtidas a partir dos sinais do enlace reverso). Em [1], esse procedimento é 
utilizado para estabelecer uma função-custo, cuja maximização aproxima uma solução ótima global em uma rede cooperativa, sem a necessidade de comunicação entre células. A partir dessa função-custo, é obtido o algoritmo do gradiente estocástico com restrições (CSG). O CSG é um algoritmo de baixa complexidade computacional, com boas características de convergência [1].

O desempenho do arranjo adaptativo pode ser avaliado através da função de distribuição de probabilidade da SINR (signal-to-interference-plus-noise ratio), denotada por $F(\gamma)$, requerendo considerar-se um amplo conjunto de condições de operação. Ou seja, faz-se necessário levar em conta um grande número de combinações de ângulos de chegada para o sinal do enlace reverso e os sinais interferentes co-canal. Assim, a avaliação de desempenho do algoritmo requer um número muito grande de simulações, considerando, além das muitas combinações de ângulos de chegada, numerosas realizações do algoritmo, requeridas para se obter os valores médios. Nesse caso, torna-se importante dispor de um modelo apropriado que descreva o comportamento do algoritmo adaptativo, permitindo obter essa informação com um custo computacional muito menor, utilizando agora expressões analíticas ao invés de simulações. Assim, neste trabalho, são propostos modelos analíticos para o momento de primeira ordem do vetor de coeficientes do arranjo adaptativo e para a curva de SINR. Esses modelos são derivados assumindo passos de adaptação pequenos, condição esta em que o sistema apresenta uma convergência suave mesmo para uma única realização.

Este artigo está organizado como segue. Na Seção II, são discutidas as características do algoritmo CSG. A Seção III apresenta o modelo que descreve o comportamento dos coeficientes do algoritmo e como ele é utilizado para determinar uma expressão analítica da SINR. Na Seção IV, visando avaliar a qualidade do modelo derivado, são confrontados os resultados obtidos por simulação Monte Carlo e pelo modelo proposto. Finalmente, na Seção V, são apresentadas as conclusões finais deste trabalho.

\section{Algoritmo CSG}

O diagrama de blocos de um arranjo adaptativo de antenas é mostrado na Fig. 1, no qual o sinal de enlace direto é dividido em $M$ ramos. Cada um desses sinais é multiplicado pelo seu respectivo coeficiente complexo $w_{i}(n)$ com $i=1, \ldots, M$. O objetivo do algoritmo adaptativo é ajustar esses coeficientes, visando aumentar a potência irradiada na direção do terminal móvel da própria célula e diminuir a potência do sinal transmitido a terminais móveis co-canal das células vizinhas. Para alcançar tais objetivos, o algoritmo usa as informações fornecidas pelo sinal do enlace reverso $\mathbf{s}(n)$ e do sinal interferente $\mathbf{u}(n)$. Em [1], é proposta como lei de operação maximizar a seguinte função-custo:

$$
\xi=\frac{\mathbf{w}^{\mathrm{H}} \mathbf{R}_{\mathrm{s}} \mathbf{w}}{\mathbf{w}^{\mathrm{H}}\left(\mathbf{R}_{\mathrm{u}}+\alpha \mathbf{I}\right) \mathbf{w}}
$$

onde $\mathbf{w}=\left[w_{1}, \ldots, w_{M}\right]^{\mathrm{T}}$ denota $\mathrm{o}$ vetor de coeficientes (considerado fixo), $\mathbf{R}_{\mathrm{s}}$ é a matriz de covariância do sinal do enlace reverso, $\mathbf{R}_{\mathrm{u}}$ é a matriz de covariância do sinal interferente e $\alpha$ é um parâmetro que estabelece o equilíbrio entre os dois objetivos mencionados anteriormente. $\mathrm{O}$ algoritmo CSG, em [1], é obtido a partir de uma interpretação intuitiva de (1). Assim, utilizando o algoritmo de descida mais íngreme (steepest descent algorithm) para a potência do sinal interferente e o algoritmo de subida mais íngreme (steepest ascent algorithm) para o sinal do enlace reverso, consegue-se maximizar (1). Entretanto, essa estratégia pode resultar em uma baixa velocidade de convergência quando a atualização dos coeficientes fizer com que os termos do numerador e do denominador de (1), simultaneamente, aumentem (ou diminuam).

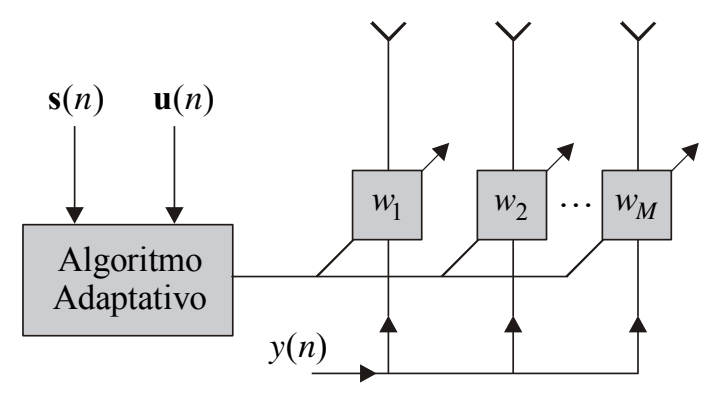

Fig. 1. Diagrama de blocos de um arranjo adaptativo de antenas.

Uma solução heurística para tal problema é utilizar um esquema de atualização em dois estágios. No primeiro estágio, o numerador de (1) é adaptado mantendo o denominador fixo; no segundo, o denominador é adaptado, mantendo o numerador fixo. Matematicamente, esse processo pode ser implementado incorporando uma matriz de projeções na equação de atualização. Assim, para o caso de dois interferentes, as equações de atualização dos coeficientes são, conforme [1], dadas por

$$
\begin{gathered}
\mathbf{v}_{1}(n)=\mathbf{w}(n)+\mu_{\mathrm{s}}\left[\mathbf{I}-\frac{\hat{\mathbf{R}}_{\mathrm{u}_{1}}(n)}{\left\|\mathbf{u}_{1}(n)\right\|^{2}}-\frac{\hat{\mathbf{R}}_{\mathrm{u}_{2}}(n)}{\left\|\mathbf{u}_{2}(n)\right\|^{2}}\right] \hat{\mathbf{R}}_{\mathrm{s}}(n) \mathbf{w}(n) \\
\mathbf{v}_{2}(n)=\mathbf{v}_{1}(n)-\mu_{\mathrm{u}}\left[\mathbf{I}-\frac{\hat{\mathbf{R}}_{\mathrm{s}}(n)}{\|\mathbf{s}(n)\|^{2}}\right]\left[\hat{\mathbf{R}}_{\mathrm{u}_{1}}(n)+\hat{\mathbf{R}}_{\mathrm{u}_{2}}(n)\right] \mathbf{w}(n) \\
\mathbf{w}(n+1)=\frac{\mathbf{v}_{2}(n)}{\left\|\mathbf{v}_{2}(n)\right\|}
\end{gathered}
$$

onde $\hat{\mathbf{R}}_{\mathrm{s}}=\mathbf{s}(n) \mathbf{s}^{\mathrm{H}}(n)$ e $\hat{\mathbf{R}}_{\mathrm{u}_{k}}=\mathbf{u}_{k}(n) \mathbf{u}_{k}^{\mathrm{H}}(n)$. Em (2) e (3), $\mu_{\mathrm{s}}$ e $\mu_{u}$ são os passos de adaptação. $O$ vetor de coeficientes é normalizado a cada atualização, mantendo o termo $\alpha \mathbf{w}^{\mathrm{H}} \mathbf{I w}$ em (1) invariante. 


\section{Modelagem EstatísticA do Algoritmo CSG}

\section{A. Momento de Primeira Ordem do Vetor de Coeficientes}

O primeiro passo para se obter o modelo que descreve o comportamento dos coeficientes é aplicar a operação valor esperado em (4). Assim,

$$
E[\mathbf{w}(n+1)]=E\left[\frac{\mathbf{v}_{2}(n)}{\left\|\mathbf{v}_{2}(n)\right\|}\right] .
$$

O cálculo do valor esperado (5) não é uma tarefa trivial. Porém, para tal, pode-se utilizar uma das seguintes aproximações:

$$
E[\mathbf{w}(n+1)] \approx \frac{E\left[\mathbf{v}_{2}(n)\right]}{E\left[\left\|\mathbf{v}_{2}(n)\right\|\right]}
$$

ou

$$
E[\mathbf{w}(n+1)] \approx \frac{E\left[\mathbf{v}_{2}(n)\right]}{\left\|E\left[\mathbf{v}_{2}(n)\right]\right\|} .
$$

A aproximação (7) garante a condição $\|E[\mathbf{w}(n)]\|=1$; entretanto, não é o caso de (6). Como o algoritmo sempre mantém a condição $\|\mathbf{w}(n)\|=1$, é importante que o modelo também satisfaça tal característica; portanto, nessa condição, é preferível considerar (7). Então, tomando o valor esperado de ambos os lados das expressões (2) e (3), obtêm-se

$$
\begin{gathered}
E\left[\mathbf{v}_{1}(n)\right]=E[\mathbf{w}(n)]+\mu_{\mathrm{s}}\left(\mathbf{I}-\mathbf{R}_{\mathrm{u}_{1}}^{\mathbf{N}}-\mathbf{R}_{\mathrm{u}_{2}}^{\mathbf{N}}\right) \mathbf{R}_{\mathrm{s}} E[\mathbf{w}(n)] \\
\mathrm{e} \\
E\left[\mathbf{v}_{2}(n)\right]=E\left[\mathbf{v}_{1}(n)\right]-\mu_{\mathrm{u}}\left(\mathbf{I}-\mathbf{R}_{\mathrm{s}}^{\mathrm{N}}\right)\left(\mathbf{R}_{\mathrm{u}_{1}}+\mathbf{R}_{\mathrm{u}_{2}}\right) E[\mathbf{w}(n)] \\
\text { onde } \quad \mathbf{R}_{\mathrm{s}}^{\mathbf{N}}=E\left[\frac{\mathbf{s}(n) \mathbf{s}^{\mathrm{H}}(n)}{\mathbf{s}^{\mathrm{H}}(n) \mathbf{s}(n)}\right] \text { e } \quad \mathbf{R}_{\mathrm{u}_{k}}^{\mathbf{N}}=E\left[\frac{\mathbf{u}_{k}(n) \mathbf{u}_{k}{ }^{\mathrm{H}}(n)}{\mathbf{u}_{k}^{\mathrm{H}}(n) \mathbf{u}_{k}(n)}\right] \text { são }
\end{gathered}
$$
matrizes de covariância normalizadas. Para o cálculo dessas matrizes, algumas simplificações, disponíveis na literatura [6], [7], podem ser utilizadas. Dentre elas, pode-se destacar o Princípio da Média [7]. No entanto, dada a quantidade reduzida de coeficientes, os valores esperados envolvendo tais matrizes devem ser calculados sem considerar quaisquer simplificações a fim de manter uma precisão adequada do modelo. Um procedimento de cálculo exato dessas matrizes é apresentado como apêndice neste trabalho. Para determinar (8) e (9), é considerado que o vetor de coeficientes e os vetores dos sinais de chegada ao arranjo são independentes. Essa consideração é justificável para passos de adaptação pequenos [8], condição que resulta em maiores valores de SINR. Substituindo (8) em (9) e a expressão resultante em (7), obtém-se a expressão final para o momento de primeira ordem do vetor de coeficientes. Então,

$$
E[\mathbf{w}(n+1)]=\frac{\mathbf{A} E[\mathbf{w}(n)]}{\sqrt{E\left[\mathbf{w}^{\mathrm{H}}(n)\right] \mathbf{B} E[\mathbf{w}(n)]}}
$$

onde $\mathbf{A}=\left[\mathbf{I}+\mu_{\mathrm{s}}\left(\mathbf{I}-\mathbf{R}_{\mathrm{u}_{1}}^{\mathrm{N}}-\mathbf{R}_{\mathrm{u}_{2}}^{\mathrm{N}}\right) \mathbf{R}_{\mathrm{s}}-\mu_{\mathrm{u}}\left(\mathbf{I}-\mathbf{R}_{\mathrm{s}}^{\mathrm{N}}\right)\left(\mathbf{R}_{\mathrm{u}_{1}}+\mathbf{R}_{\mathrm{u}_{2}}\right)\right]$ e $\mathbf{B}=\mathbf{A}^{\mathrm{H}} \mathbf{A}$.

\section{B. Razão Sinal/Ruído-mais-interferência (SINR)}

Maximizar a SINR é o objetivo fundamental do arranjo adaptativo no problema aqui tratado e, portanto, o comportamento da SINR é um importante indicador do desempenho do algoritmo adaptativo. Por definição, a SINR é dada por

$$
\gamma=\frac{P_{\mathrm{s}}}{P_{\mathrm{u}}+P_{\eta}}
$$

onde $P_{\mathrm{s}}$ é a potência (média) do sinal útil, $P_{\mathrm{u}}$ é a potência do sinal interferente e $P_{\eta}$ é a potência do ruído. Uma expressão para determinar a evolução da SINR é dada por

$$
\gamma(n)=\frac{E\left[\mathbf{w}^{\mathrm{H}}(n) \mathbf{s}(n) \mathbf{s}^{\mathrm{H}}(n) \mathbf{w}(n)\right]}{E\left[\mathbf{w}^{\mathrm{H}}(n) \mathbf{u}(n) \mathbf{u}^{\mathrm{H}}(n) \mathbf{w}(n)\right]+\sigma_{\eta}^{2} E\left[\mathbf{w}^{\mathrm{H}}(n) \mathbf{I} \mathbf{w}(n)\right]} .
$$

A expressão (12) pode ser reescrita como

$$
\gamma(n)=\frac{\operatorname{tr}\left[\mathbf{R}_{\mathrm{s}} \mathbf{K}(n)\right]}{\operatorname{tr}\left[\mathbf{R}_{\mathrm{u}} \mathbf{K}(n)\right]+\sigma_{\eta}^{2} \operatorname{tr}[\mathbf{K}(n)]}
$$

onde $\mathbf{K}(n)=E\left[\mathbf{w}(n) \mathbf{w}^{\mathrm{H}}(n)\right]$ é o momento de segunda ordem do vetor de coeficientes. Considerando que $\operatorname{tr}[\mathbf{K}(n)]=$ $E\left[\|\mathbf{w}(n)\|^{2}\right]=1$ devido à normalização dos coeficientes do algoritmo e, também, normalizando a potência do ruído, (13) resulta em

$$
\gamma(n)=\frac{\rho_{\mathrm{S}} \operatorname{tr}\left[\mathbf{R}_{\mathrm{s}} \mathbf{K}(n)\right]}{\rho_{\mathrm{u}} \operatorname{tr}\left[\mathbf{R}_{\mathrm{u}} \mathbf{K}(n)\right]+1}
$$

onde $\rho_{\mathrm{s}}$ é o fator de normalização da potência do sinal do terminal móvel da própria célula e $\rho_{u}$ é o fator de normalização da potência do sinal interferente. De (14), conclui-se que conhecendo o comportamento do momento de segunda ordem do vetor de coeficientes pode-se também conhecer o comportamento da SINR.

Uma das características do algoritmo CSG é a pouca variabilidade obtida entre diferentes realizações. Assim sendo, o modelo que descreva o comportamento do algoritmo deve ser simples o suficiente para justificar sua aplicação ao invés de simulações Monte Carlo. Visando a simplicidade do modelo, a seguinte aproximação é considerada:

$$
E[\mathbf{K}(n)]=E\left[\mathbf{w}(n) \mathbf{w}^{\mathrm{H}}(n)\right] \approx E[\mathbf{w}(n)] E\left[\mathbf{w}^{\mathrm{H}}(n)\right] .
$$

Tal aproximação é bastante utilizada na análise do algoritmo LMS, apresentando resultados aceitáveis [9], [10], sendo válida para passos de adaptação pequenos. Assim, o comportamento da SINR é completamente determinado a partir do momento de primeira ordem do vetor de coeficientes dado por (10).

\section{Resultados de Simulação}

Nesta seção, são apresentados alguns resultados de simulação obtidos pelo método de Monte Carlo (MC) e pelo modelo proposto. Os resultados para comparação são separados em três grupos: comportamento da função de distribuição de probabilidades da SINR $[F(\gamma)$ em regime 
permanente], comportamento de convergência de $\gamma(n)$ e comportamento de convergência dos coeficientes. Em toda esta seção, são consideradas duas fontes interferentes e as matrizes de covariância são obtidas utilizando o modelo de sinal apresentado em [1].

\section{A. Comportamento de $F(\gamma)$ em Regime Permanente}

Para o enlace reverso, assume-se que o ângulo de chegada do sinal do terminal móvel da própria célula $\theta_{\mathrm{s}}$ e os ângulos de chegada dos sinais interferentes, $\theta_{\mathrm{u}_{1}}$ e $\theta_{\mathrm{u}_{2}}$, são tomados em valores discretos (uniformemente espaçados quantizados a cada $\left.4^{\circ}\right)$ dos conjuntos $\Theta_{\mathrm{s}}=\left(-60^{\circ}, 60^{\circ}\right), \Theta_{\mathrm{u}_{1}}=\left(-36^{\circ},-12^{\circ}\right)$ e $\Theta_{\mathrm{u}_{2}}=\left(12^{\circ}, 36^{\circ}\right)$, respectivamente. Para cada combinação de ângulo de chegada, duas matrizes $\mathbf{K}$ em regime permanente são determinadas, uma por simulação $\mathrm{MC}$ e outra utilizando o modelo proposto. As matrizes $\mathbf{K}$ obtidas são substituídas em (14), obtendo assim as correspondentes SINR. O conjunto resultante de SINR é utilizado para calcular as suas estatísticas segundo o método proposto em [1], tanto para os resultados obtidos por simulação MC (200 realizações independentes) quanto para os obtidos pelo modelo proposto. São consideradas quatro situações resultantes da combinação de dois valores de abertura de ângulo de chegada, $\Delta_{\mathrm{s}}=\Delta_{\mathrm{u}}=3^{\circ}$ e $30^{\circ}$, e dois valores para o número de elementos por arranjo, $M=4$ e $M=8$. O conjunto de parâmetros para os quatro casos é: $I=12$ caminhos de desvanecimento independentes, fatores de potência normalizados $\rho_{\mathrm{s}}=36 \mathrm{~dB}$ e $\rho_{\mathrm{u}}=15 \mathrm{~dB}$ e passos de adaptação $\mu_{\mathrm{s}}=\mu_{\mathrm{u}}=0,01$. Todos esses parâmetros são selecionados visando reproduzir situações práticas em ambientes urbanos. A Fig. 2 mostra os resultados obtidos nas quatro situações descritas, permitindo verificar-se a predição muito boa obtida pelo modelo.

\section{B. Comportamento da Convergência de $\gamma(n)$}

Para avaliar o comportamento de $\gamma(n)$, são consideradas as mesmas quatro situações descritas anteriormente. Porém, é selecionada apenas uma combinação de ângulos de chegada $\theta_{\mathrm{s}}, \theta_{\mathrm{u}_{1}}$ e $\theta_{\mathrm{u}_{2}}$. Também o valor do passo de adaptação é modificado, visando obter-se o maior valor de SINR em regime permanente para a combinação de ângulos considerada. Os demais parâmetros são mantidos. A Fig. 3(a) mostra os resultados de simulações $\mathrm{MC}$ e do modelo proposto para valores de abertura de ângulo de chegada igual a $\Delta_{\mathrm{s}}=\Delta_{\mathrm{u}}=3^{\circ}$ para $M=4$ e $M=8$. Para o caso de $M=4$, é utilizado $\mu_{\mathrm{s}}=\mu_{\mathrm{u}}=0,05$ e, para $M=8$, é considerado $\mu_{\mathrm{s}}=\mu_{\mathrm{u}}=0,01$. A combinação de ângulos de chegada selecionados são: $\theta_{\mathrm{s}}=60^{\circ}, \theta_{\mathrm{u}_{1}}=-16^{\circ}$ e $\theta_{\mathrm{u}_{2}}=36^{\circ}$. A Fig. 3(b) apresenta os resultados para $\Delta_{\mathrm{S}}=\Delta_{\mathrm{u}}=30^{\circ}$, considerando novamente duas situações: $M=4$ e $M=8$. Para o caso de $M=4$, é utilizado $\mu_{\mathrm{s}}=\mu_{\mathrm{u}}=0,01$ e, para $M=8, \mu_{\mathrm{s}}=\mu_{\mathrm{u}}=0,007$. Percebe-se também neste exemplo um casamento muito bom entre simulação e modelo proposto.

\section{Comportamento dos Coeficientes}

Para avaliar o desempenho do modelo dos vetores de coeficientes, são consideradas as duas situações descritas na Fig. 3(a), mostrando, em cada caso, os resultados de simulação MC e do modelo proposto tanto para a parte real quanto para a parte imaginária dos coeficientes. Todos esses resultados podem ser verificados na Fig. 4, constatando-se um bom casamento em ambas as regiões transiente e de regime permanente.

\section{CONCLUSÕES FinAis}

Modelos analíticos para o momento de primeira ordem do vetor de coeficientes e para a curva de SINR foram apresentados, considerando a hipótese de adaptação lenta. Em geral, foi observado um bom casamento entre o comportamento descrito pelas simulações e o obtido através do modelo de predição tanto em regime transiente quanto em regime permanente. Assim, com o emprego das expressões determinísticas do modelo proposto, pode-se avaliar o comportamento de arranjos adaptativos para aplicações em sistemas celulares de uma forma mais rápida, direta e segura do que a obtida através de simulações.

\section{APÊNDICE \\ CÁlCUlo EXATo dAs MATRIZES DE COVARIÂNCIA NORMALIZADAS}

A metodologia de cálculo das matrizes de covariância normalizadas $\mathbf{R}_{\mathbf{x}}^{\mathrm{N}}$ é desenvolvida considerando um vetor de sinais Gaussianos complexos $\mathbf{x}(n)$, com matriz de covariância R. A expressão da matriz de covariância normalizada é

$$
\mathbf{R}_{\mathbf{x}}^{\mathrm{N}}=E\left[\frac{\mathbf{x}(n) \mathbf{x}^{\mathrm{H}}(n)}{\mathbf{x}^{\mathrm{H}}(n) \mathbf{x}(n)}\right] .
$$

Para determinar o valor esperado em (A.16), é definida uma função matricial auxiliar $\mathbf{F}(\omega)$, cujos elementos são dados pela seguinte expressão [11]:

$$
f_{i, j}(\omega)=\frac{1}{\pi^{M} \operatorname{det}(\mathbf{R})} \underbrace{\int_{-\infty}^{\infty} \cdots \int_{-\infty}^{\infty} \frac{x_{i} x_{j}}{\mathbf{x}^{\mathrm{H}} \mathbf{x}}}_{M \text { vezes }} \mathrm{e}^{-\mathbf{x}^{\mathrm{H}} \mathbf{L}^{-1} \mathbf{x}} d \mathbf{x}
$$

onde $\mathbf{L}^{-1}=\mathbf{R}^{-1}+\omega \mathbf{I}$, tal que

$$
E\left[\frac{\mathbf{x}(n) \mathbf{x}^{\mathrm{H}}(n)}{\mathbf{x}^{\mathrm{H}}(n) \mathbf{x}(n)}\right]=\mathbf{F}(0) .
$$

Diferenciando (A.17) em relação a $\omega$ e utilizando as propriedades da função densidade de probabilidade Gaussiana complexa, obtém-se

$$
\left\{E\left[\frac{\mathbf{x}(n) \mathbf{x}^{\mathrm{H}}(n)}{\mathbf{x}^{\mathrm{H}}(n) \mathbf{x}(n)}\right]\right\}_{i, j}=\int_{0}^{\infty} \frac{\left\{\mathbf{R}[\mathbf{I}+\omega \mathbf{R}]^{-1}\right\}_{i, j}}{\operatorname{det}(\mathbf{I}+\omega \mathbf{R})} d \omega .
$$




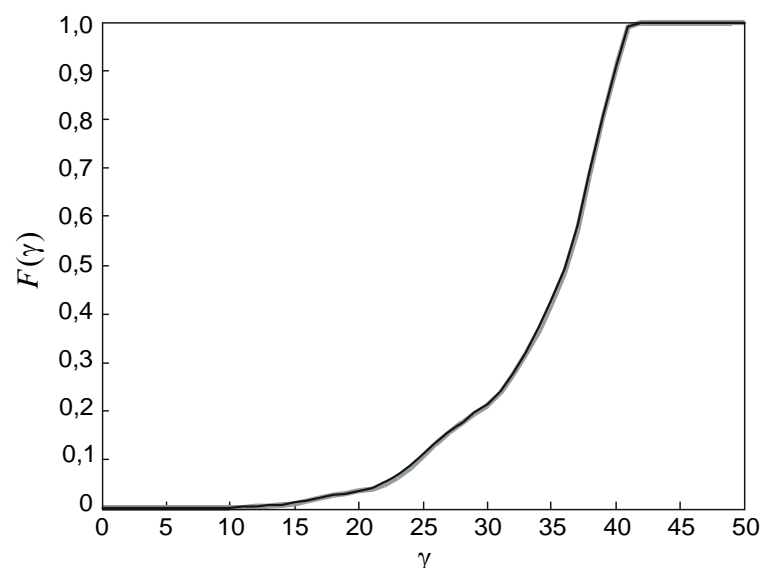

(a)

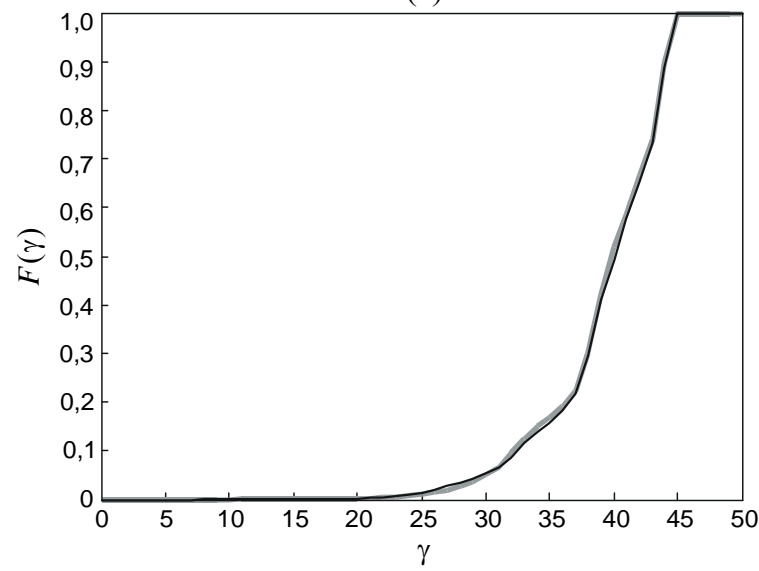

(c)

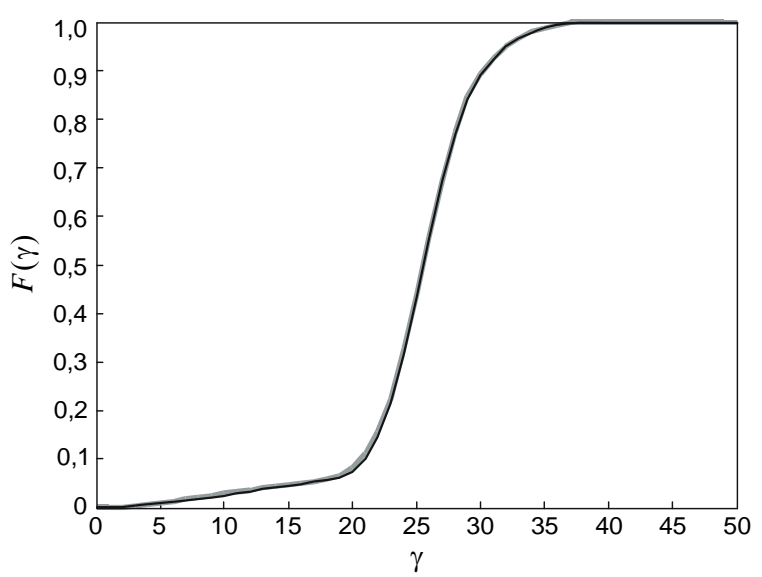

(b)

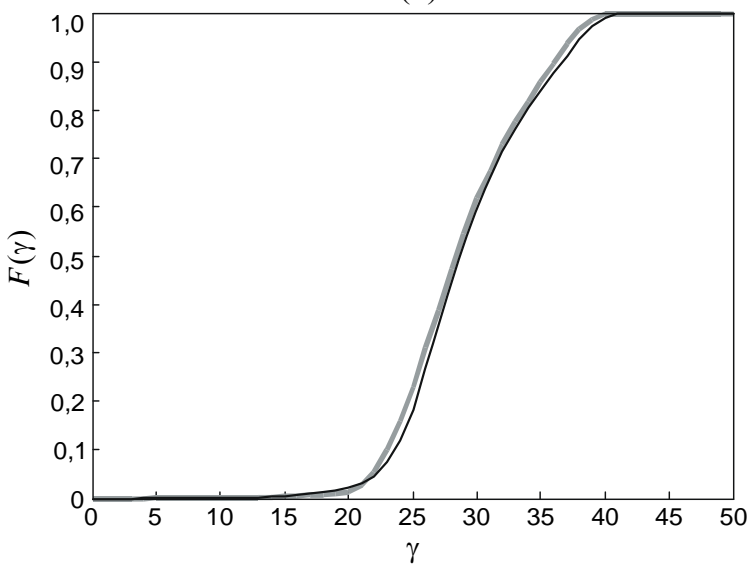

(d)

Fig. 2. Comportamento da função distribuição de probabilidade da SINR. Simulação MC (linha cinza); modelo proposto (linha preta). (a) CDF para quatro elementos e $\Delta_{\mathrm{s}}=\Delta_{\mathrm{u}}=3^{\circ}$. (b) CDF para quatro elementos e $\Delta_{\mathrm{s}}=\Delta_{\mathrm{u}}=30^{\circ}$. (c) CDF para oito elementos e $\Delta_{\mathrm{s}}=\Delta_{\mathrm{u}}=3^{\circ}$ (d) CDF para oito elementos e $\Delta_{\mathrm{s}}=\Delta_{\mathrm{u}}=30^{\circ}$.

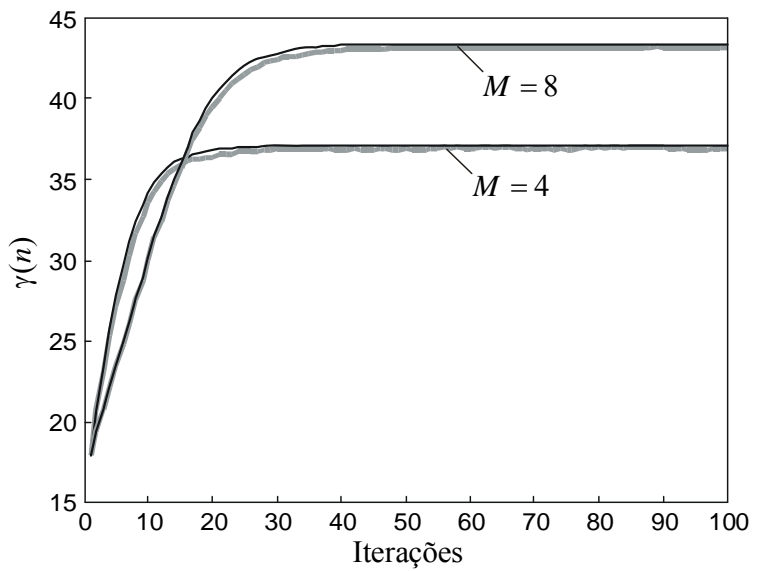

(a)

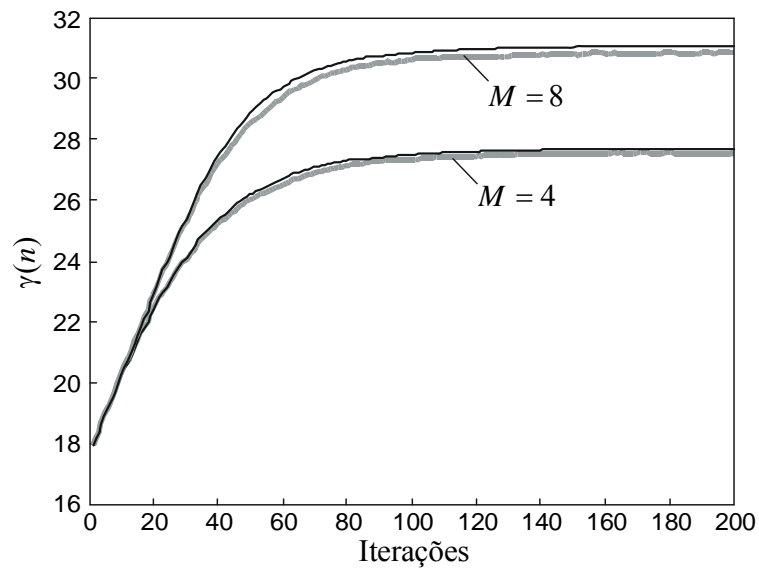

(b)

Fig. 3. Comportamento de convergência de $\gamma(n)$. Simulação MC (linha cinza); modelo proposto (linha preta). (a) Comportamento para $\Delta_{\mathrm{s}}=\Delta_{\mathrm{u}}=3^{\circ}$. (b) Comportamento para $\Delta_{\mathrm{s}}=\Delta_{\mathrm{u}}=30^{\circ}$.

Utilizando a decomposição $\mathbf{R}=\mathbf{Q} \mathbf{\Lambda} \mathbf{Q}^{\mathrm{T}}$, onde $\mathbf{Q}$ é a matriz de autovetores de $\mathbf{R}$ e $\boldsymbol{\Lambda}$ é uma matriz diagonal contendo os autovalores $\lambda_{i}$, para $i=1,2, \ldots, M$, de $\mathbf{R}$, obtém-se

$$
E\left[\frac{\mathbf{x}(n) \mathbf{x}^{\mathrm{H}}(n)}{\mathbf{x}^{\mathrm{H}}(n) \mathbf{x}(n)}\right]=\mathbf{Q H} \mathbf{Q}^{\mathrm{T}} .
$$

Assim, o valor esperado (A.16) é determinando a partir dos $M$ elementos da matriz diagonal $\mathbf{H}$, dados por 


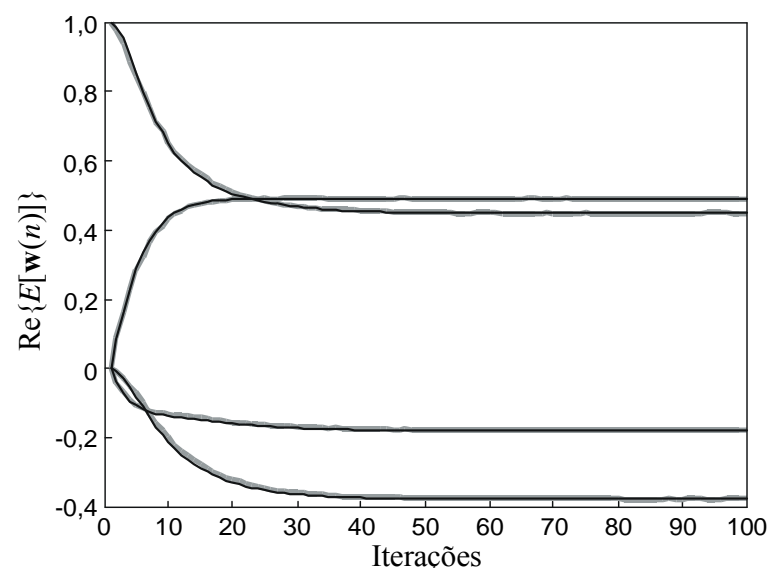

(a)

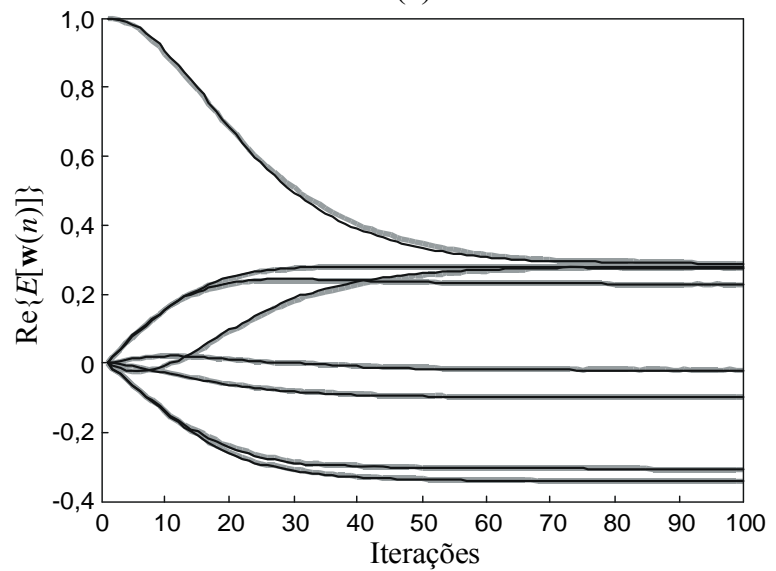

(c)

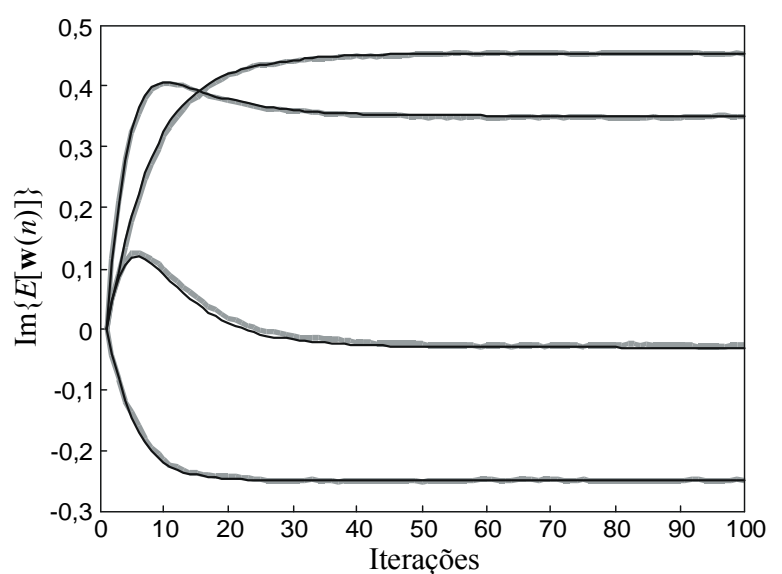

(b)

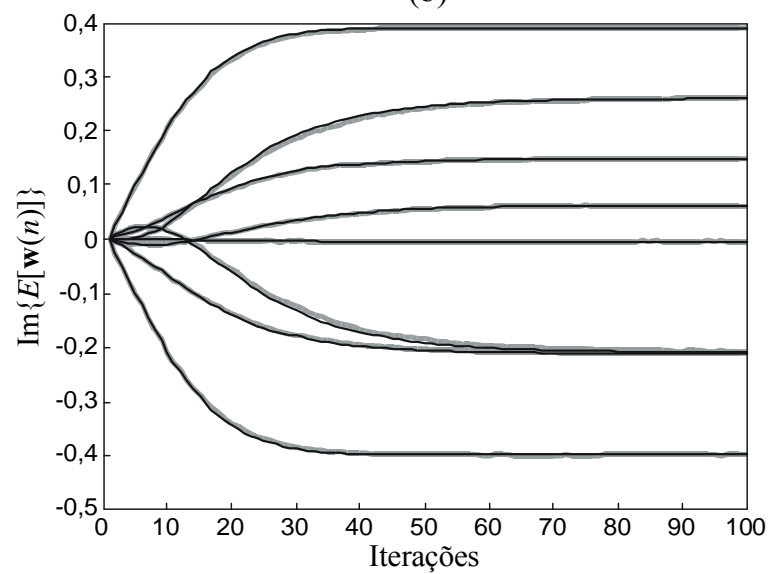

(d)

Fig. 4. Comportamento dos coeficientes. Simulação MC (linha cinza); modelo proposto (linha preta). Situação $M=4$ : (a) Parte real dos coeficientes, (b) Parte imaginária dos coeficientes. Situação $M=8$ : (c) Parte real dos coeficientes, (d) Parte imaginária dos coeficientes.

A integral (A.21) pode ser calculada utilizando uma expansão em frações parciais. Como exemplo, considere-se o caso em que todos os autovalores são diferentes, situação comum nesta aplicação, resultando na seguinte expressão para os elementos de $\mathbf{H}$ :

$$
h_{k}=A_{1, k} \lambda_{k}+A_{2, k} \ln \left(\lambda_{k}\right)+\sum_{\substack{i=1 \\ i \neq k}}^{M} B_{i, k} \ln \left(\lambda_{i}\right)
$$

onde

$$
A_{1, k}=\lambda_{k}^{M-2} / \prod_{\substack{i=1 \\ i \neq k}}^{M}\left(\lambda_{k}-\lambda_{i}\right), \quad B_{i, k}=\frac{\lambda_{k} \lambda_{i}^{M-1}}{\left(\lambda_{i}-\lambda_{k}\right) \prod_{\substack{j=1 \\ j \neq i}}^{M}\left(\lambda_{i}-\lambda_{j}\right)}
$$

e

$$
A_{2, k}=-A_{1, k} \lambda_{k} \sum_{\substack{i=1 \\ i \neq k}}^{M}\left[\lambda_{i} /\left(\lambda_{k}-\lambda_{i}\right)\right]
$$

\section{REFERÊNCIAS}

[1] D. R. Morgan, "Downlink adaptive array algorithms for cellular mobile communications," IEEE Trans. Commun., vol. 51, no. 3, pp. 476-488, Mar. 2003.

[2] L. C. Godara, "Cellular Systems," Handbook in Antennas in Wireless Communications, L.C. Godara, ed., CRC Press, 2002.
[3] J. M. Goldberg and J. R. Fonollosa, "Downlink beamforming for spatially distributed sources in cellular mobile communications," Signal Processing, vol. 65, no. 2, pp. 181-197, 1998.

[4] B. M. Hochwald and T. L. Marzetta, "Adapting a downlink array from uplink measurements," IEEE Trans. Signal Process., vol. 49, no. 3, pp. 642-653, Mar. 2001.

[5] Y-C. Liang and F. P .S. Chin, "Downlink channel covariance matrix (DCCM) estimation and its applications in wireless DS-CDMA systems," IEEE J. Select. Areas Commun., vol. 9, no. 2, pp. 222-232, Feb. 2001.

[6] M. H. Costa and J. C. M. Bermudez, "An improved model for the normalized LMS algorithm with Gaussian inputs and large number of coefficients," in Proc. IEEE Int. Conf. Acoust., Speech, and Signal Processing, Orlando, USA, vol. II, May 2002, pp. 1385-1388.

[7] E. M. Lobato, O. J. Tobias, and R. Seara, "Stochastic modeling of the transform domain $\varepsilon L M S$ algorithm for a time-varying environment," in Proc. XIII European Signal Processing Conference (EUSIPCO 2005), Antalya, Turkey, Sept. 2005, pp. 1-4.

[8] B. Farhang-Boroujeny, Adaptive Filters: Theory and Applications, John Wiley \& Sons, 1998.

[9] J. E. Kolodziej, J. Tobias, and R. Seara, "Stochastic model for the NSAF algorithm considering slow adaptation and Gaussian data," in Proc. International Telecommunications Symposium (ITS 2006), Fortaleza, Brazil, Sept. 2006, pp. 166-170.

[10] N. J. Bershad, P. Celka, and J. M. Vesin, "Stochastic analysis of gradient adaptive identification of nonlinear systems with memory for Gaussian data and noisy input and output measurements," IEEE Trans. Signal Process., vol. 47, no. 3, pp. 675-689, Mar. 1999.

[11] M. Rupp, "The behavior of LMS and NLMS algorithms in the presence of spherically invariant processes," IEEE Trans. Signal Process., vol. 41, no. 3, pp. 1149-1160, Mar. 1993. 